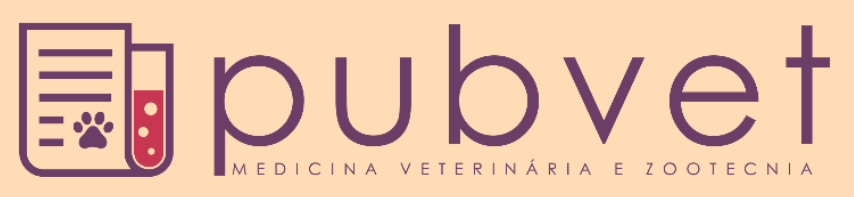

https://doi.org/10.31533/pubvet.v13n9a415.1-6

\title{
Terapia neural no tratamento do megaesôfago congênito em cão: relato de caso
}

\author{
Bruna Aparecida Lima Gonçalves ${ }^{1 * \bullet}$, Leonardo Rocha Vianna ${ }^{2}$, Camila de Castro Andrade ${ }^{3 \bullet}$ \\ ${ }^{I}$ Médica Veterinária do Centro de Estudos em clínica e cirurgia de animais (CECCA) da Pontifícia Universidade Católica de \\ Minas Gerais Betim, Mestranda UFMG, acupunturista, terapeuta neural. \\ ${ }^{2}$ Médico Veterinário formado na UFMG, Mestre em clínica e cirurgia pela UFMG, acupunturista, terapeuta neural, medico \\ chinês, Ten. Coronel médico veterinário reformado da PMMG e especialista em medicina natural. \\ ${ }^{3}$ Médica veterinária residente do Centro de Estudos em clínica e cirurgia de animais (CECCA) da Pontifícia Universidade \\ Católica de Minas Gerais Betim \\ *Autor para correspondência. E-mail: bruna_alg@yahoo.com.br
}

\begin{abstract}
Resumo. A Terapia Neural (TN) teve início em 1905 com a descoberta do primeiro anestésico sintético, a procaína. As bases científicas da Terapia Neural se iniciaram com os russos Pavlov e Speransky posteriormente com os irmãos alemães Huneke, também tem importância Pischinger e Hans H. Reckeweg dentre outros. É praticada na Áustria, Alemanha, Argentina, Brasil, Canada, Chile, Colômbia, Costa Rica, Cuba, Espanha, Estados Unidos, México, Suíça, Turquia e alguns outros países. Consiste em uma terapia reguladora que busca ativar os mecanismos autorreguladores do organismo, por meio da aplicação de anestésicos locais em baixas concentrações em determinadas regiões corporais, como pontos de acupuntura, cicatrizes, articulações, intravenosa, em gânglios entre outras. O objetivo deste trabalho foi demonstrar a efetividade da Terapia Neural em um animal com quadro clínico de megaesôfago congênito.
\end{abstract}

Palavras chaves: terapia neural, megaesôfago, procaína

\section{Neural therapy in the treatment of congenital megaesophagus in dog: case report}

\begin{abstract}
Neural Therapy (NT) began in 1905 with the discovey of the first syntetic anesthetic, the procain. The Neural Therapy Scientific base began with the russians Pavlov and Speransky, later, with the German brothers Heneke, and also Pischinger and Hans H. Reckeweg have importance, among others. It is practiced in Austria, Germany, Argentina, Brazil, Canada, Chile, Colombia, Costa Rica, Cuba, Spain, United States, Mexico, Switzerland, Turkey and some another country. It consists in a regulatory therapy that seeks to activate the body self-regulating mechanism through the apliccation of local anesthetics in low concentrations in certain body regions, like acupuncture points, scars, articulations, intravenous, ganglia, among others. This study aims to demonstrate the effectiveness of Neural Therapy in an animal with congenital mesaesophagus.
\end{abstract}

Keywords: neural therapy, megaesophagus, procaine

\section{Terapia neural en el tratamiento del megaesófago congénito en perros: reporte de un caso}

Resumen. La Terapia Neural (TN) comenzó en 1905 con el descubrimiento del primer anestésico sintético, la procaína. La base científica de la Terapia Neural comenzó con los rusos Pavlov y Speransky, y más tarde con los hermanos alemanes Huneke, también tiene 
importancia Pischinger y Hans H. Reckeweg entre otros. Se practica en Austria, Alemania, Argentina, Brasil, Canadá, Chile, Colombia, Costa Rica, Cuba, España, Estados Unidos, México, Suiza, Turquía y algunos otros países. Consiste en una terapia reguladora que busca activar los mecanismos autorreguladores del cuerpo mediante la aplicación de anestésicos locales a bajas concentraciones en ciertas regiones del cuerpo, como puntos de acupuntura, cicatrices, articulaciones, intravenosas, ganglios, entre otros. El objetivo de este estudio fue demostrar la efectividad de la Terapia Neural en un animal con megaesófago congénito.

Palabras clave: terapia neural, megaesófago, procaína

\section{Introdução}

Megaesôfago é o distúrbio de motilidade considerado como a principal causa de regurgitação em cães (Ettinger, 2003; Washabau, 2003). Tal enfermidade pode ser congênita ou adquirida, que por sua vez é subdividida em idiopática e secundária. A hipomotilidade esofágica resulta em acúmulo de conteúdo no esôfago, gerando dilatação e consequentemente a sintomatologia clínica associada (Mace et al., 2012; Martín, 2012; Torres, 1997). Em um panorama geral, a prevalência de casos de megaesôfago em cães é significativa em raças como Shar Pei, Pastor Alemão, Schnauzers miniatura, Labrador e Samoieda (Alves et al., 2013; Gaynor et al., 1997; Mace et al., 2012). A etiologia do megaesôfago congênito não é totalmente compreendida, porém sabe-se que está associada a um defeito na inervação vagal aferente do esôfago (Quintavalla et al., 2017). A forma adquirida, por sua vez, possui diversas etiologias, sendo a mais importante a miastenia grave (Washabau, 2003).

Os cães acometidos podem apresentar desde apenas regurgitação de alimento não digerido, esta pode ocorrer de minutos a horas após a ingestão, até pneumonia por aspiração, sendo a principal complicação e causa de mortalidade nos pacientes que apresentam o distúrbio em questão. Os sinais clínicos no megaesôfago congênito geralmente se apresentam em filhotes logo após o desmame, e é comum que estes animais sejam menos desenvolvidos em comparação aos demais da ninhada. A dilatação do esôfago nestes casos é generalizada (Tanaka et al., 2010).

O diagnóstico é realizado através dos sinais clínicos, radiografias comuns e contrastadas (esofagografia) e endoscopia (Quessada, 1993). Pelas radiografias comuns é possível visualizar acúmulos de líquido e alimento ao longo da extensão do esôfago, ao passo que, com auxílio do sulfato de bário como contraste, pode-se avaliar o órgão como um todo, seu grau de dilatação e sua motilidade (Torres, 1997).

O tratamento do megaesôfago adquirido ou secundário consiste na correção da causa base, o tratamento das formas congênita e adquirida idiopática normalmente é conservativo e embasado no manejo nutricional do paciente. A indicação é que o animal deve ser alimentado em posição vertical, para que a gravidade ajude no trânsito da ingesta até o estômago. O alimento (de consistência variável, pastoso ou sólido) deve ter alto valor calórico e dividido em pequenas refeições mais frequentes (Washabau, 2003). Em caso de pneumonias por aspiração, a antibioticoterapia de largo espectro deve ser instituída (Ettinger, 2003; Fossum, 2014). As opções farmacológicas (drogas pró-cinéticas como a cisaprida e a metoclopramida) são limitadas e de pouco benefício para cães (Ettinger, 2003; Fossum, 2014). Há relatos de tratamentos bem-sucedidos com o uso do sildenafil (Quintavalla et al., 2017) e de cura através da medicina tradicional chinesa e acupuntura (Martín, 2012).

Os casos de megaesôfago congênito possuem prognóstico reservado (Martín, 2012), devido à natureza deste distúrbio, complicações e a falta de um tratamento definitivo. Embora a precocidade do diagnóstico auxilie no tratamento, a expectativa de vida destes pacientes infelizmente é pequena, e estimada em cerca de um ano de idade (Alves et al., 2013; Mace et al., 2012). Os animais que apresentam a enfermidade em sua forma adquirida idiopática compartilham deste mesmo prognóstico. Todavia, o megaesôfago adquirido ou secundário possui prognóstico mais favorável (Washabau, 2003).

A Terapia Neural faz parte das medicinas biológicas e terapias reguladoras que buscam ativar os mecanismos autorreguladores do organismo. É um estímulo mediante injeções de anestésico local sem vasoconstritor em pontos específicos, tanto a quantidade quanto a duração do efeito anestésico são secundários, sabe se que o efeito terapêutico sobressai ao efeito anestésico (Fischer, 2006; Gonçalves et al., 2019). A Terapia Neural faz parte da medicina complementar de origem relativamente recente. Suas 
bases teóricas fazem parte da fisiologia russa de meados do século XIX, pesquisas foram desenvolvidas por Pavlov, Speransky, entre outros. Posteriormente, na primeira metade do século XX os irmãos alemães Walter e Ferdinand Huneke, aprofundaram na pesquisa e na prática da Terapia Neural (Toscano \& Pinilla, 2012). É uma terapia integral e tem indicação em várias enfermidades: reumáticas, neurológicas, ginecológicas, amigdalites, sinusites, otites, asma, afecções dermatológicas, entre outras (De La Torre \& Bonilla, 2012; Gonçalves et al., 2019). Consiste na utilização de anestésicos locais (lidocaína e procaína) com baixas concentrações de 0,1 a $1 \%$, em partes do corpo escolhidas, conforme o distúrbio (Cruz \& Naffah, 2011). As aplicações podem ser intradérmicas, subcutâneas, endovenosa, intra-arterial, em gânglios nervosos, entre outras. A quantidade e o local de aplicação são escolhidos pelo médico veterinário após fazer o levantamento pelo histórico de vida do paciente das áreas afetadas. Estas podem ser em feridas cirúrgicas, cicatrizes, pontos de acupuntura, pontos gatilhos, entre outras (Burrel, 2008; Cruz \& Naffah, 2011; Gonçalves et al., 2019; Vianna \& Gonçalves, 2017).

Um dos objetivos da Terapia Neural é descobrir "campos interferentes" no corpo do paciente, que são áreas onde as células estão afetadas, fora do estado fisiológico, onde não há polarização e repolarização celular (parabiose ou estado de hibernação celular). A administração de anestésicos locais sobre essas células restabelece o potencial elétrico (Cruz \& Naffah, 2011), ou seja, visa eliminar "campos interferentes" e devolver ao organismo seu potencial de autorregulação (Álvarez-Urbay et al., 2007). Um anestésico local diluído tem alto potencial repolarizante, geralmente cloridrato de procaína $(1 \%, 0,75 \%, 0,5 \%, 0,35 \%)$ que estabiliza o potencial de membrana celular e de todo o sistema nervoso (Burrel, 2008). A aplicação pode ser diretamente no local afetado, doloroso, como: inserções de ligamentos, periósteo, tendão, articulações, nervos periféricos, cápsulas articulares, pontos de acupuntura, entre outros (Castro, 2011; Fischer, 2006; Gonçalves et al., 2019; Vianna \& Gonçalves, 2017). Segundo Bravo-Monsalvo et al. (2008) a dose máxima do cloridrato de procaína a 0,7\% quando utilizada de forma intradermal é de $7 \mathrm{mg} / \mathrm{kg}$, em um estudo em cães com dermatite atópica utilizou se também a dose endovenosa de $0,1 \mathrm{mg} / \mathrm{kg}$. A procaína é hidrolisada em ácido p-animobenzóico (PABA) e dietilaminoetanol (DEAE), sendo rapidamente absorvida após a administração intramuscular e subcutânea. A distribuição é influenciada pelo metabolismo, a procaína é rapidamente hidrolisada, não se acumula nos tecidos e plasma, se liga pouco a proteínas. É metabolizada no plasma pelas colinesterases. O PABA aparece $80,90 \%$ conjugado na urina, a DEAE também aparece na urina 33,3\% inalterada, o restante é degradado em outros metabólitos (Bulcão et al., 2011). O tempo médio de degradação é de 7 minutos e máximo de 20 minutos (Castro, 2011; Fischer, 2006).

Este artigo objetiva relatar o caso de um cão diagnosticado com megaesôfago congênito, cuja terapia baseada em manejo alimentar e terapia neural resultaram em uma excelente recuperação, com desaparecimento do quadro clínico e da dilatação esofágica avaliada por raio x comum e contrastado.

\section{Relato de caso}

Cão, SRD, filhote de aproximadamente 45 dias de idade, proveniente de uma ninhada de uma cadela de rua, foi adotado pelos tutores e após 1 semana de sua adoção foi levado para atendimento ao Centro de Estudos em Clínica e Cirurgia em Animais da Pontifícia Universidade Católica de Minas Gerais (CECCA - PUC Minas) Figura 1. A principal queixa era vômitos constantes após as refeições, os quais ocorriam geralmente decorridos poucos minutos após a ingestão dos alimentos. Pelo diálogo com os tutores foi esclarecido que se tratava na verdade de regurgitação, uma vez que o cão expelia o alimento não digerido. Houve episódios de expulsão de líquido através das narinas. Como consequência da dificuldade de se alimentar, o animal também apresentava prostração há dois dias. Foi adequadamente vermifugado quando adotado.

Ao exame físico, notou-se que o animal estava magro, porém os parâmetros vitais estavam dentro da normalidade. Cada respiração, era visível uma dilatação sutil no terço médio do pescoço, na face ventral. De acordo com a suspeita principal de megaesôfago congênito, o paciente foi encaminhado para radiografia, comum primeiramente, e logo em seguida contrastada, utilizando como contraste o sulfato de bário. A radiografia comum mostrou deslocamento ventral da traqueia, bem como o acúmulo de ar na porção cervical do esôfago. Figura 2. Com o auxílio do contraste, o diagnóstico de megaesôfago congênito foi fechado, graças à visualização da dilatação generalizada do órgão e confirmada pelos exames de imagem. Figura 3. 


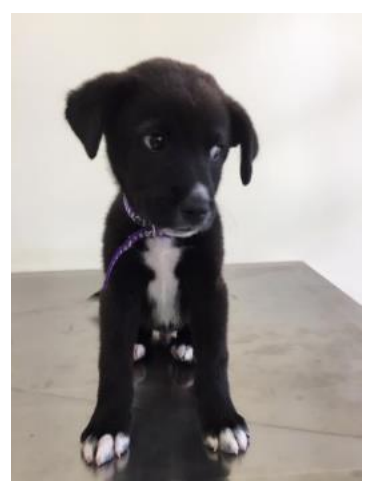

Figura 1. Animal no primeiro dia de tratamento

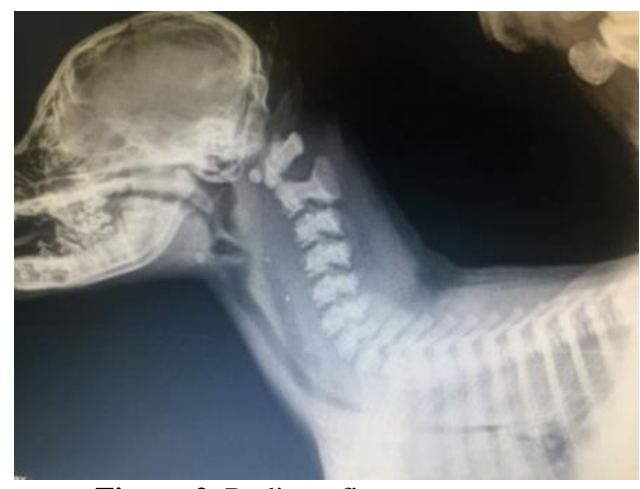

Figura 2. Radiografia sem contraste.

O paciente foi internado para que pudesse ser observado e voltasse a se alimentar espontaneamente sem regurgitar, ainda no ambiente hospitalar foi realizado o manejo alimentar adequado. Os tutores foram orientados a alimentar o animal em posição vertical, dividindo a quantidade diária de alimento, de consistência pastosa, em diversas pequenas porções. Foi prescrito antibiótico de amplo espectro (amoxicilina + clavulanato de potássio, na dose de 12,5 mg/kg BID por 7 dias), como profilaxia para um acometimento pulmonar secundário.

No retorno, nove dias após a primeira consulta, o filhote ainda apresentava regurgitações, embora em menor frequência. Foi então mudado o tipo de alimento fornecido para rações com grãos maiores, para que o tempo gasto pelo animal em suas refeições aumentasse. Além disso, foi iniciada a terapia neural.

Ao longo do tratamento, o animal ganhou peso e cessou completamente as regurgitações. Cerca de 5 meses após o início da terapia neural, foram realizadas novas radiografias para acompanhamento do caso clínico. Não foram visualizadas alterações anatômicas na radiografia, o contraste mostrou o esôfago sem dilatação alguma. Figura 4. Desta forma, pode-se afirmar que a cura foi alcançada neste caso de megaesôfago.

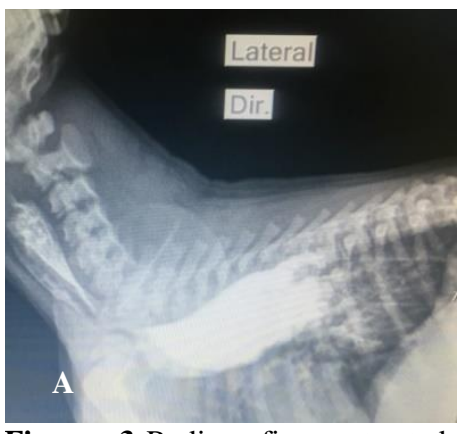

Figuras 3 Radiografias contrastadas Evidência de o megaesôfago.

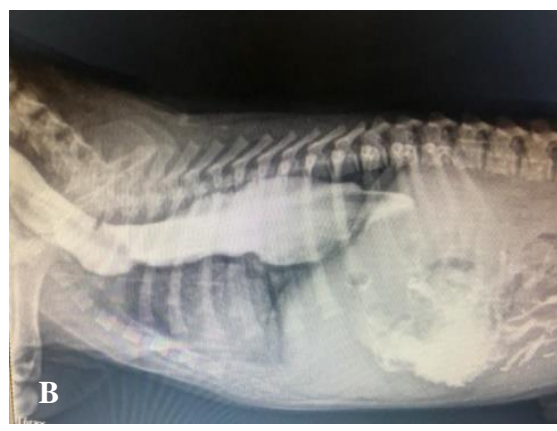

antes do tratamento com Terapia Neural.

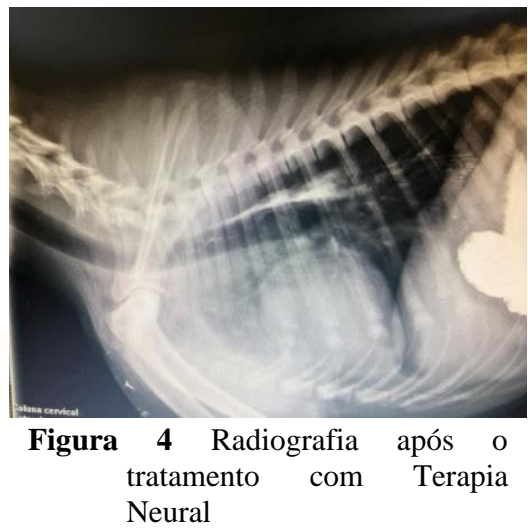

As aplicações foram locais de forma subcutânea na região ventral do pescoço em 4 pontos $(2 \mathrm{em}$ cada lado) com aplicação de $0,5 \mathrm{ml}$ em cada ponto de cloridrato de procaína a 0,7\%. Figura 6.

O tratamento realizado consistiu em quatro sessões de Terapia Neural com intervalo de 21 dias. Paciente atualmente está pesando $26 \mathrm{~kg}$. Figura 7.

\section{Discussão}

Os sinais clínicos apresentados pelo paciente estão em acordo com os descritos na literatura, sendo o mais importante à regurgitação (Gaynor et al., 1997; Mace et al., 2012; Martín, 2012; Tanaka et al., 2010; Washabau, 2003). O diagnóstico definitivo foi obtido pelas radiografias comuns e contrastadas, cujos achados são condizentes com os descritos por Torres (1997). Embora houvesse relatos de expulsão de líquido pelas narinas, felizmente o paciente não adquiriu pneumonia por aspiração, sendo a antibioticoterapia instituída apenas profilática. 


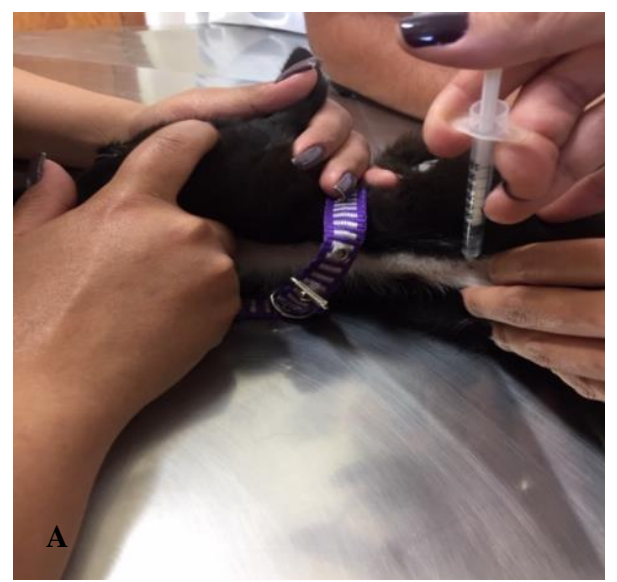

Figuras 6. Aplicações subcutâneas de cloridrato de procaína a 0,7\%. Região ventral do pescoço.

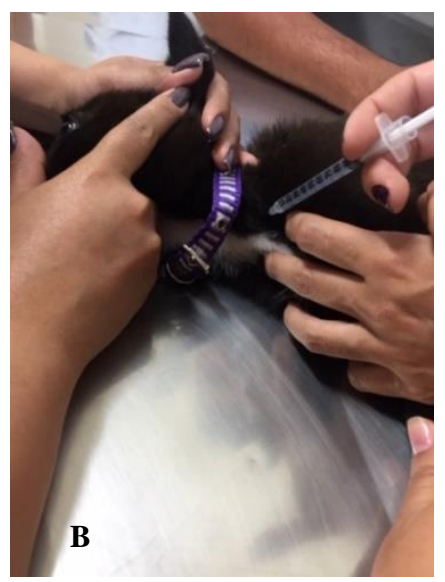

(1)

.

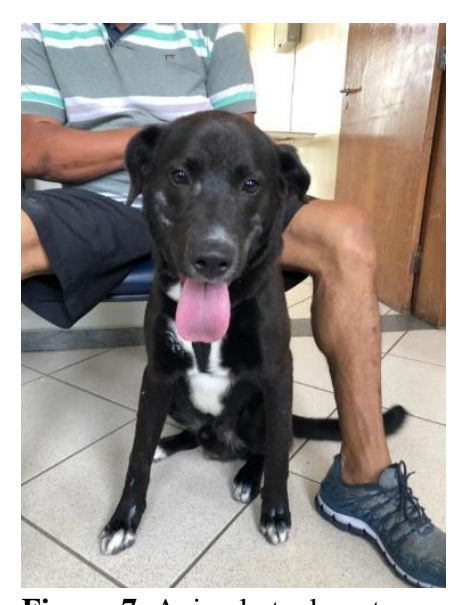

Figura 7. Animal atualmente com 9 meses.

O manejo nutricional do animal é a chave do tratamento do megaesôfago congênito, uma vez que não há até o momento terapias eficazes para a correção da motilidade esofágica (Tanaka et al., 2010). De modo geral, este manejo consiste em utilizar a gravidade como aliada, evitando que a ingesta se acumule no órgão dilatado (Mace et al., 2012; Martín, 2012). Washabau (2003) enfatiza que além da alimentação em posição vertical com pequenas refeições, a consistência do alimento fornecido deve ser adaptada a cada paciente. No caso relatado, foram utilizadas dietas pastosas e sólidas, as quais foram mais bem toleradas pelo animal. De acordo com Diamant et al. (1974), o manejo nutricional adequado é de extrema importância em cães com megaesôfago congênito, ao passo que controla os sinais clínicos e produz bem-estar, enquanto a motilidade esofágica pode se recuperar com o tempo. Os autores salientam que, na espécie canina, a maturação da inervação e musculatura esofágicas pode perdurar até 12 meses de idade. Este pode ter sido um fator que auxiliou na recuperação do paciente no caso relatado.

O tratamento foi monitorado por constantes retornos, com exames físicos do paciente e conversas com os tutores. Após cinco meses, repetição das radiografias comum e com contraste. Estas auxiliam na avaliação da dilatação esofágica e dos pulmões, importante quando se considera que os pacientes que apresentam megaesôfago são muito suscetíveis à pneumonia por aspiração (Mace et al., 2012). Neste relato de caso, a cura foi constatada através da radiografia contrastada, na qual não mais se visualizou alterações do esôfago.

Ressalta-se o papel das terapias complementares no tratamento do megaesôfago congênito. Esse caso mostrou que a Terapia Neural é um método complementar de tratamento, que apresentou bons resultados. O intervalo entre as sessões é individualizado, de acordo com cada caso (Gonçalves et al., 2019).

\section{Considerações finais}

Pelo caso relatado, pode-se demonstrar a importância da Terapia Neural como uma maneira de tratamento no quadro de megaesôfago. É uma doença de grande importância na medicina veterinária devido à dificuldade de tratamento. Muitos tutores desistem do tratamento devido considerar o manejo muito complexo. E muitas vezes, os animais são descartados ou eutanasiados. Por ser um tratamento longo e difícil, muitas vezes com muitas tentativas e erros até alcançar a abordagem ideal, o engajamento dos tutores é fundamental. Neste caso relatado, pode-se perceber que a dedicação dos tutores em seguir as orientações e a disciplina quanto às sessões de terapia neural, foram determinantes para que o animal alcançasse a cura clínica. A Terapia Neural ainda é pouco conhecida no Brasil, mas os resultados são impressionantes.

\section{Referências bibliográficas}

Álvarez-Urbay, M. A., Alvarez, H. F. C., Ávila, M. B., Murgas, G. F. \& Álvarez, J. S. (2007). Resultados de la terapia neural en las faringitis crónicas. Revista Archivo Médico de Camagüey, 11(2):0-0.

Alves, N. M., Dias, T. P., Pereira, A. M., Rosito, J. \& Borges, M. M. (2013). Megaesôfago congênito em cão. PUBVET, 72327-2445. 
Bravo-Monsalvo, A., Vázquez-Chagoyán, J., Gutiérrez, L. \& Sumano, H. (2008). Clinical efficacy of neural therapy for the treatment of atopic dermatitis in dogs. Acta Veterinaria Hungarica, 56(4):459469.

Bulcão, R. P., Arbo, M. D., Rohers, M., Paniz, C., Cervi, F., L., Thiesen, F. V., . . Garcia, S. C. (2011). Procaína: Efeitos farmacológicos e toxicológicos. Revista de Ciências Farmacêuticas Básica e Aplicada, 32(3):297-303.

Burrel, S. E. (2008). Salud, enfermaria y terapia neural.

Castro, R. A. (2011). Bases para la terapia neural em caninos y felinos. Buenos Aires, Argentina: Dunken.

Cruz, Y. \& Naffah, R. F. (2011). Microtúbulos y terapia neural: propuesta de una investigación promisoria. Revista Med de la Facultad de Medicina, 19(1):82-92.

De La Torre, F. T. \& Bonilla, L. B. P. (2012). Los principios de la terapia neural desde los fundamentos del nervismo hasta la neurociencia actual. Revista de la Universidad Industrial de Santander, 44(2):57-65.

Diamant, N., Szczepanski, M. \& Mui, H. (1974). Idiopathic megaesophagus in the dog: reasons for spontaneous improvement and a possible method of medical therapy. The Canadian Veterinary Journal, 15(3):66-71.

Ettinger, S. N. (2003). Principles of treatment for canine lymphoma. Clinical Techniques in Small Animal Practice, 18(2):92-97.

Fischer, L. (2006). Terapia neural según Huneke fundamentos técnicas, aplicación práctica. México: Hippokrates.

Fossum, T. W. (2014). Cirurgia de pequenos animais (4 ed. Vol. 1). São Paulo: Elsevier Brasil.

Gaynor, A. R., Shofer, F. S. \& Washabau, R. J. (1997). Risk factors for acquired megaesophagus in dogs. Journal of the American Veterinary Medical Association, 211(11):1406-1412.

Gonçalves, B. A. L., Vianna, L. R., Fernandes, A. L., Teixeira, A. C. B. \& Amaral, K. P. (2019). Tratamento com Terapia Neural em cão com sequela de cinomose: Relato de caso. PUBVET, 13(7):1-6.

Mace, S., Shelton, G. D. \& Eddlestone, S. (2012). Megaesophagus. Compendium, 34(2):E1-E1.

Martín, F. M. (2012). Megaesófago en la especie canina: abordaje según los principios de la Medicina Veterinaria Tradicional China y la acupuntura. Revista Internacional de Acupuntura, 6(4):158-161.

Quessada, A. M. (1993). Surgical esophageal diseases in small animals. A review. Ciência Rural, 23(2):249-255.

Quintavalla, F., Menozzi, A., Pozzoli, C., Poli, E., Donati, P., Wyler, D. K., . . Bertini, S. (2017). Sildenafil improves clinical signs and radiographic features in dogs with congenital idiopathic megaoesophagus: a randomised controlled trial. Veterinary Record, 11-6.

Tanaka, N. M., Hoogevonink, N., Tucholski, Â. P., Trapp, S. M. \& Frehse, M. S. (2010). Megaesôfago em cães. Revista Acadêmica Ciência Animal, 8(3):271-279.

Torres, P. (1997). Megaesófago en el perro. Revisión bibliográfica y proposición de una nueva clasificación. Archivos de Medicina Veterinaria, 29(1):13-23.

Toscano, T. F. \& Pinilla, B. L. B. (2012). Los principios de la terapia neural desde los fundamentos del nervismo hasta la neurociencia actua. Revista de La Universidade Industrial de Santander, 4457-65.

Vianna, L. R. \& Gonçalves, B. A. L. (2017). Entenda a terapia neural. Revista CFMV, 7444-47.

Washabau, R. J. (2003). Gastrointestinal motility disorders and gastrointestinal prokinetic therapy. The Veterinary Clinics of North America. Small Animal Practice, 33(5):1007-1028.

Recebido: 2 de agosto de 2019 .

Aprovado: 8 de outubro, 2019.

Publicado: 24 de outubro, 2019.

Licenciamento: Este artigo é publicado na modalidade Acesso Aberto sob a licença Creative Commons Atribuição 4.0 (CC-BY 4.0), a qual permite uso irrestrito, distribuição, reprodução em qualquer meio, desde que o autor e a fonte sejam devidamente creditados. 